\title{
A rare case of Candida parapsilosis endocarditis in a young healthy woman - case report
}

\author{
Mijomir Pelemiš ${ }^{1}$, Goran Stevanović ${ }^{*}$, Lidija Lavadinović ${ }^{1}$ Snežana Matić², Ivana Milošević ${ }^{1}$, Miloš Korać1, \\ Svetislav Pelemiš ${ }^{3}$, Milan Nedeljković ${ }^{2}$ and Milica Prostran ${ }^{4}$
}

\begin{abstract}
Disseminated fungal infections are still rare conditions, mostly caused by Candida spp. during immunosuppression. Infection of immunocompetent individuals is uncommon. Endocarditis is a rare manifestation during candidaemia, mostly in patients with prosthetic valves. Affection of previously unaltered valves is uncommon.
\end{abstract}

Case presentation: We presented a case of a young, previously healthy female patient with endocarditis, caused by Candida parapsilosis. The initial symptom, fever, was present four months before hospital admittance. She was febrile without other symptoms and during observation in a local hospital. After her condition deteriorated, she was transferred to the Institute for infectious and tropical diseases, Belgrade. Clinical findings on admission include petechial skin rash and moderate hepatosplenomegaly. Newly developed systolic murmur was noted, and Candida parapsilosis was isolated in multiple blood cultures. Echocardiography revealed $15 \times 14 \mathrm{~mm}$ vegetations on the right aortic vellum.

She was treated with antifungal drugs (fluconasole, liposomal amphotericin B), and the affected valve was successfully replaced. The same strain of Candida parapsilosis was isolated from the intraoperative material of the valve.

There were no markers of immunosuppression or other conditions which could affect the immune system.

Conclusion: After a prolonged period of treatment she was successfully cured, and she received a long-term intermittent suppressive fluconasole therapy for the time being.

Keywords: Native valve, Antifungal therapy, Post surgical treatment, Candidaemia, Candida endocarditis

\section{Background}

Although disseminated fungal infections are more common today than before, they still remain rare conditions, mostly caused by Candida spp. Candida parapsilosis and Candida tropicalis are the most common causes in Europe [1,2]. Risk factors include immunosuppression (HIV, neutropenia, transplants, solid tumors), but infection of immunocompetent individuals is uncommon $[1,3]$. Candidaemia has a significant mortality rate, up to $44 \%[1,2]$.

Endocarditis is present in 5-25\% of patients with candidaemia, mostly in patients with prosthetic valves $[4,5]$. Affection of previously unaltered heart valves is

\footnotetext{
* Correspondence: goran_drste@yahoo.com

'Clinic for Infectious and Tropical Diseases, Clinical Center of Serbia, Faculty of Medicine, University of Belgrade, Bulevar oslobodjenja 16, 11000, Belgrade, Serbia

Full list of author information is available at the end of the article
}

uncommon. During the past 20 years, there have been only three cases reported in Sweden [4]. Other authors also refer to Candida endocarditis as an extremely rare occurrence in patients with normal native cardiac valves [6,7].

\section{Case presentation}

We will present a case report of a previously healthy twenty-three year old Caucasian female patient, from a higher social-culture level, living in Belgrade suburbs. She had a negative history data to preexisting diseases, drug use or any underlining conditions.

First symptom-fever, presented four months before hospital admittance, was treated with third generation cephalosporin (ceftriaxone 2,0 gr daily i.v.) and resolved within 5 days. During the next month she was febrile without other symptoms. During that time the patient was observed in a local hospital, until her condition deteriorated - with anemia, leucopenia and petechial 
skin rash, and she was transferred to the Institute for Infectious and Tropical Diseases, Belgrade, Clinical Centre of Serbia. Clinical findings on admission included discreet petechial skin rash, moderate hepatosplenomegaly, whereas other systems were unaffected, including normal heart sounds. Laboratory tests showed moderate elevation of erythrocyte sedimentation rate, intermediary anemia and leucopenia, with elevated C-reactive protein. (Table 1) Candida parapsilosis- sensitive to all systemic antifungal drugs was isolated in multiple blood cultures. The initial treatment included IV fluconazole $200 \mathrm{mg} / 12 \mathrm{~h}$. During the third day of therapy, a newly developed systolic murmur was noted, presenting an indication for echocardiography. The patient was afebrile starting from that moment.

Echocardiography revealed $15 \times 14 \mathrm{~mm}$ vegetations on the right aortic vellum. (Figure 1) As Candida endocarditis usually appears in immunosuppressive patients as well as in patients with prosthetic valves, extensive clinical investigation was performed, to uncover any possible cause of immunosuppression. During a four-week period, all possible infectious agents were tested, including HIV, HBV, HCV, EBV, Leischmania and tuberculosis. Normal absolute count and ratio of $\mathrm{CD} 4, \mathrm{CD} 2, \mathrm{CD} 3$ and $\mathrm{CD} 8$ lymphocytes was noted, as well as normal concentration of immunoglobulines and sufficient immunological functions. There were no markers of autoimmune diseases which could affect the immune system. Hematological investigation was performed, as well as abdominal and chest $\mathrm{CT}$, so the possible presence of solid tumors was excluded. A "10-panel urine screen" drug test was negative. During investigation, the patient was treated with fluconazole (200 mg/12 h, i.v.). Control echocardiography showed progressive enlargement of vegetations, spreading to the other vellum, so the treatment was continued with liposomal amphotericin B intravenously, $50 \mathrm{mg}$ daily. Since the transoesophageal echocardiography showed that after two weeks of new treatment there were two additional vegetations affecting vellums $(17 \times 6 \mathrm{~mm}$ and $12 \times 3 \mathrm{~mm}$ ), it was necessary to replace the affected heart valve. Control blood cultures, repeated daily from the fifth day of hospitalization (patient was afebrile), were sterile. Preoperatively, patient was treated with fluconazole for 38 days, and liposomal amphotericin B for 15 days. The affected valve (Figure 2) was successfully replaced, and the same strain of Candida parapsilosis was isolated from intraoperative material of the valve.

Further conservative antifungal therapy was administered after operation, until laboratory findings were normalized, five months later. During this period the patient was treated with liposomal amphotericin B for 36 days, IV in the daily dose of $50 \mathrm{mg}$. For the rest of the period she was treated with fluconazole IV $200 \mathrm{mg}$ in the dose intervals of 12 hours (approximately four months). During the whole period, no side-effects were observed.

During the 3 year follow-up, there were no other pathological developments, and the patient has been physically active and working.

\section{Discussion}

This report presents a rare disease progress in a young and previously healthy person, without any predisposing conditions - no immunodeficiency or previous valve defect [4-6]. In spite of intensive systemic antifungal therapy, which is recommended $[2,4,5]$ there was no improvement, so the operative valve replacement was necessary, which has also been reported in other cases [4-7].

After the patient was discharged from the hospital, she received a long-term intermittent suppressive fluconasole therapy. There is some difference in opinion concerning the time length of this therapy, including possibility of life-long treatment $[5,8,9]$. During the three year follow-up, our patient has been taking $200 \mathrm{mg}$ of fluconasole twice a week with complete recovery and no other physical complaints.

Table 1 Laboratory results during course of illness, and follow-up

\begin{tabular}{|c|c|c|c|c|c|c|}
\hline Analysis* & Admission day & Day 15 & Day 30 (preoperatively) & Day 40 (8 days after operation) & Discharge day & After 3 year follow-up \\
\hline SE (mm/hour) & 54 & 50 & 48 & 36 & 18 & 2 \\
\hline $\mathrm{HgB}(\mathrm{gr} / \mathrm{l})$ & 72 & 105 & 126 & 117 & 146 & 140 \\
\hline $\operatorname{Er} \times 10^{12} / 1$ & 2,71 & 3,21 & 3,66 & 3,82 & 4,12 & 4,08 \\
\hline Le $\times 10^{9} / /$ & 3,7 & 3,1 & 4,0 & 6,5 & 6,2 & 5,6 \\
\hline $\operatorname{Tr} \times 10^{9} / 1$ & 218 & 317 & 298 & 321 & 251 & 253 \\
\hline fibrinogen $(\mathrm{gr} / \mathrm{l})$ & 2,2 & 4,1 & 4,4 & 4,8 & 2,4 & 2,6 \\
\hline CRP (mg/l) & 38 & 22 & 28 & 20 & 4 & 2 \\
\hline PT (sec) & 14,5 & 13,2 & 12,9 & 16,8 & 17,8 & 18,9 \\
\hline
\end{tabular}

Legend:

*SE- erythrocytes sedimentation rate during first hour, HgB - hemoglobin, Er - ery throcytes, Le - leucocytes, Tr - thrombocytes, CRP - C-reactive protein, PT - prothrombine time. 

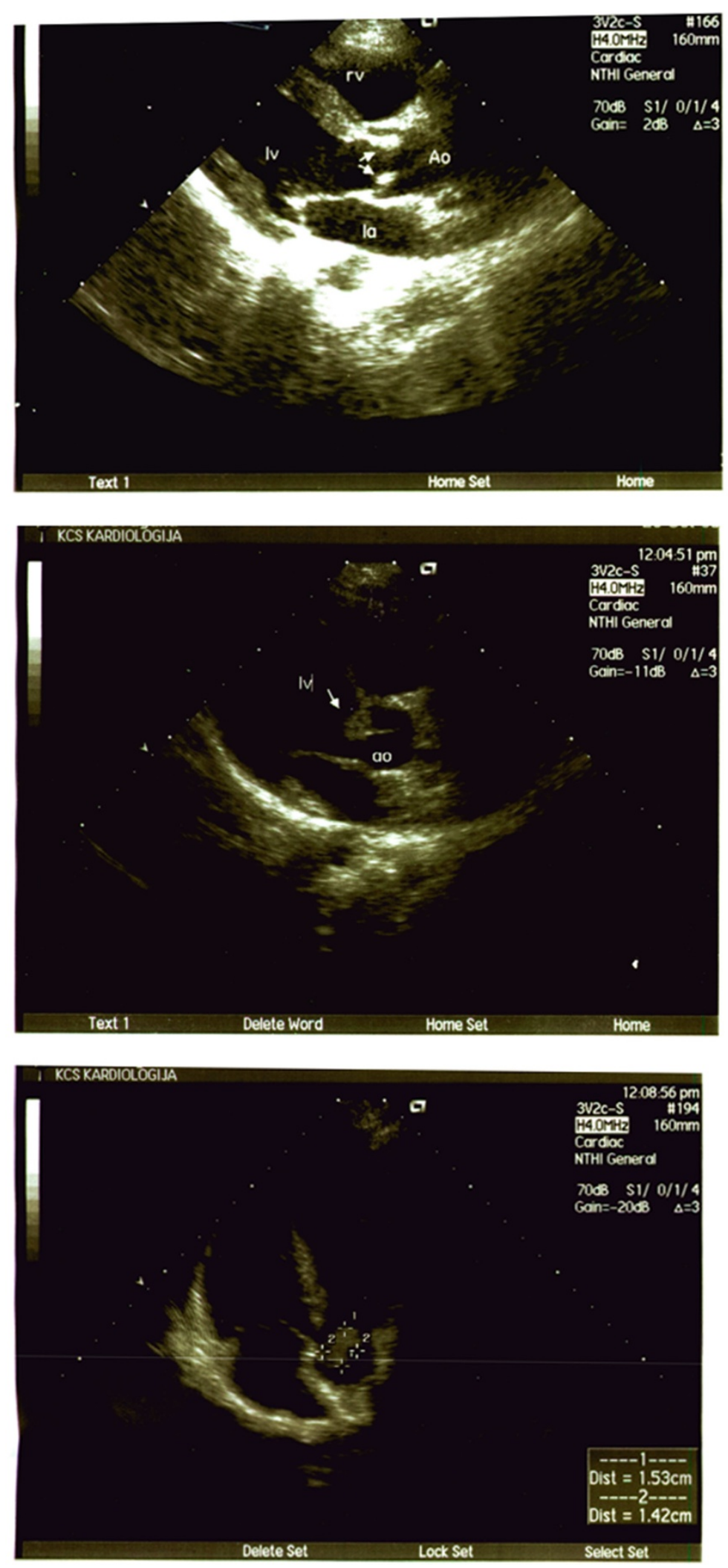

Figure 1 Transthoracal echocardiografy in longitudinal and apical 4 chamber view vegetations on the aortic valves. 


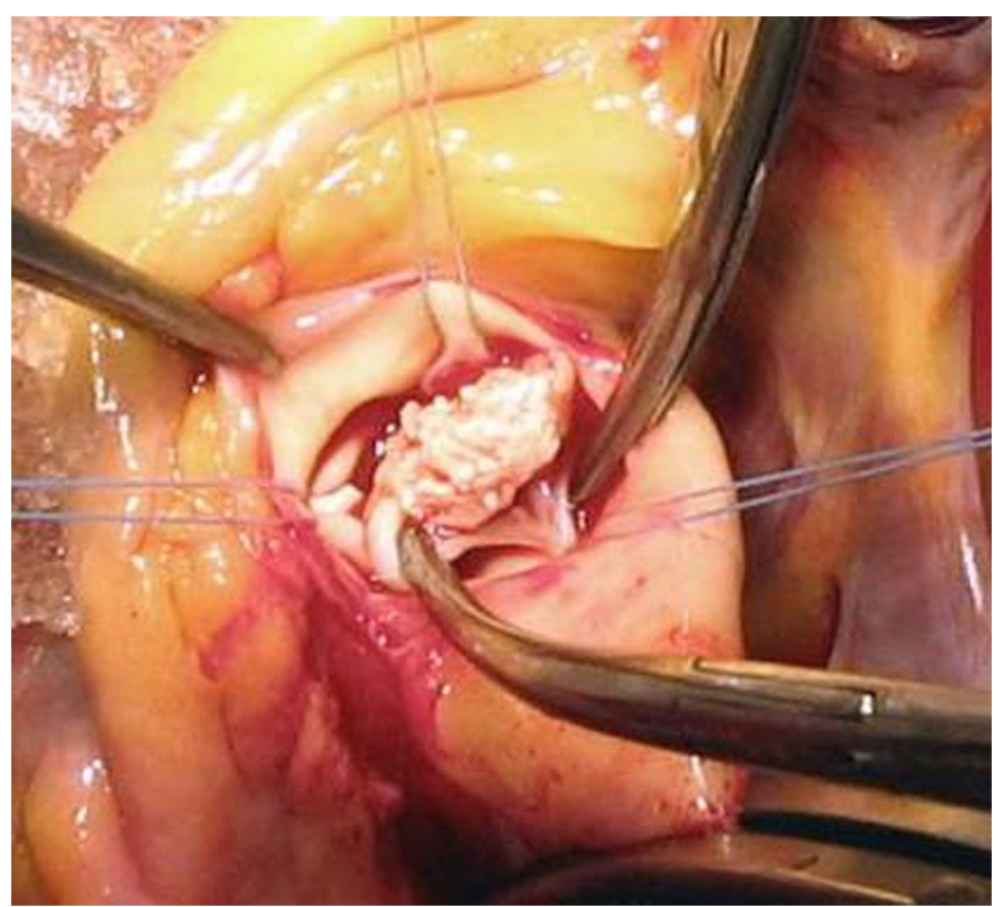

Figure 2 Intraoperaive vegetation findings - aortic valve.

\section{Conclusions}

Although rare, Candida endocarditis in previously healthy patients, with no clear risk factors, is possible. During blood cultivation, one should always look for fungus. Candida endocarditis therapy is a combination of antifungal drugs and surgery, and the use of drugs is longlasting.

\section{Consent}

Written informed consent was obtained from the patient for publication of this case report and any accompanying images. A copy of the written consent is available for review by the Editor-in-Chief of this journal.

\section{Competing interests}

The authors declare that they have no competing interests.

\section{Authors' contributions}

PM was the primary treating physician and is responsible for the treatment: SG participated in the treatment of patients and manuscript preparation; LL participated in the treatment of patients and manuscript preparation; MS was the main treating cardiologist who was constantly monitoring the patient; Ml assisted in the treatment and monitoring of the patient and helped to draft the manuscript; KM assisted in the treatment and monitoring of the patient and helped to draft the manuscript; PS assisted in the treatment and monitoring of the patient and helped to draft manuscript; NM was the chief cardiologist responsible for echocardiogaphy and preoperative preparation; PM was the clinical pharmacologist responsible for the selection of antifungal drugs and their combination, as well as for the monitoring of adverse effects. All authors read and approved the final form manuscript.

\section{Acknowledgement}

The Authors wish to acknowledge Milica Jovanovic MD, PhD, microbiologist, who did an excellent job of identifying the causative agent.

\section{Author details}

${ }^{1}$ Clinic for Infectious and Tropical Diseases, Clinical Center of Serbia, Faculty of Medicine, University of Belgrade, Bulevar oslobodjenja 16, 11000, Belgrade, Serbia. ${ }^{2}$ Clinic for Cardiology, Clinical Center of Serbia, Dr Koste Todorovica 8, 11000, Belgrade, Serbia. ${ }^{3}$ Clinic for Otorhinolaringology, Clinical Center of Serbia, Pasterova 2, 11000, Belgrade, Serbia. ${ }^{4}$ Department of Pharmacology, Clinical Pharmacology and Toxicology, Faculty of Medicine, University of Belgrade, 11000, Belgrade, Serbia.

Received: 14 November 2012 Accepted: 19 February 2013 Published: 22 February 2013

\section{References}

1. Marchetti O, Bille J, Fluckiger U, Eggiman P, Ruef C, Garbino J, Calandra T, Glausser MP, Tauber MG, Pittet D: Epidemiology of candidemia in Swiss tertiary care hospitals: secular trends, 1991-2000. Clin Infect Dis 2004, 38:311-320,

2. Pfaller MA, Diekema DJ, Jones RN, Sader HS, Fluit AC, Hollis RJ, Messer SA, and SENTRY Participant Group: International surveillance of bloodstream infections due to Candida species: frequency of occurrence and in vitro susceptibilities to fluconazole, ravuconazole, and voriconazole of isolates collected from 1997 through 1999 in the SENTRY Antimicrobial Surveillance Program. J Clin Microbiol 2001, 39:3254-3259.

3. Almirante B, Rodríguez D, Park BJ, et al: Epidemiology and Predictors of Mortality in Cases of Candida Bloodstream Infection: Results from Population-Based Surveillance, Barcelona, Spain, from 2002 to 2003. J Clin Microbiol 2005, 43(4):1829-1835.

4. Hogevik H, Alestig K: Fungal endocarditis - a report on seven cases and a brief review. Infection 1996, 24:17-21.

5. Branco L, Pitta ML, Bernardes $L$, et al: A review of infectious endocarditis due to Candida [Abstract]. Rev Port Cardiol 1997, 16:967-974.

6. Gullu AU, Akcar M, Arnaz A, et al: Candida parapsilosis tricuspid native valve endocarditis: 3-year follow-up after surgical treatment. Interact Cardiovasc Thorac Surg 2008, 7:513-514. 
7. Kan CD, Luo CY, Lin PY, et al: Native-valve endocarditis due to Candida parapsilosis. Interact Cardiovasc Thorac Surg 2002, 1:66-68.

8. Zedtwitz-Liebenstein $\mathrm{K}$, Gabriel $\mathrm{H}$, Willinger B, et al: Prosthetic valve endocarditis due to Candida tropicalis complicated by multiple pseudoaneurysms. Infection 2001, 29:177-179.

9. Chim CS, Ho PL, Yuen ST, Yuen KY: Fungal endocarditis in bone marrow transplantation: case report and review of literature. J Infect 1998, 37:287-291.

doi:10.1186/1749-8090-8-29

Cite this article as: Pelemiš et al:: A rare case of Candida parapsilosis endocarditis in a young healthy woman - case report. Journal of Cardiothoracic Surgery 2013 8:29.

\section{Submit your next manuscript to BioMed Central and take full advantage of:}

- Convenient online submission

- Thorough peer review

- No space constraints or color figure charges

- Immediate publication on acceptance

- Inclusion in PubMed, CAS, Scopus and Google Scholar

- Research which is freely available for redistribution 